American Journal of Applied Sciences 9 (4): 535-541, 2012

ISSN 1546-9239

(C) 2012 Science Publications

\title{
A New Strategy for Signaling Overhead Reduction in the Proxy Mobile IPv6 Protocol
}

\author{
Adnan J. Jabir, S. Shamala and Z. Zuriati \\ Department of Communication Technology and Networks, \\ Faculty of Computer Science and Information Technology, \\ Universiti Putra Malaysia 43400 Serdang, Selangor, Malaysia
}

\begin{abstract}
Problem statement: The rapid growth of the Internet usage has been realized by the Mobile IPv6 (MIPv6). Concurrent to these technological advances is the communication overheads. Proxy Mobile IPv6 (PMIPv6) has been standardized to overcome the long handoff latency, packet loss and signaling overhead of MIPv6 and to exempt the mobile node from any involvement in the handoff process. Although the PMIPv6 has resolved the related MIPv6 problems, it incurs a long handoff time due to the frequent binding updates which requires multiple and redundant authentications and registration operations. For these reasons, research on PMIPv6 has already been devoted to reduce the handoff latency and the packet loss ratio by pre-registering the mobile node to the new network. However, these researches have not been able to reduce the number of signals required for authentication and registration processes which are frequently performed during the binding updates. Approach: In this study, an enhanced PMIPv6 signaling strategy known as the I-PMIPv6 protocol is proposed, in which the authentication and the registration signaling are integrated and deployed coherently to reduce the handoff signaling cost. Results: The proposed protocol is evaluated analytically and the numerical results show that the I-PMIPv6 outperforms the PMIPv6 in terms of the handoff latency time. Conclusion: Reducing the signaling overhead by integrating the authentication and registration steps has indeed reduced the handoff latency which leads to reduce the service disruption during the host movement.
\end{abstract}

Key words: Internet Engineering Task Force (IETF), Local Mobility Anchor (LMA), Mobile Access Gateway (MAG), Proxy Mobile IPv6 (PMIPv6), Home Agent (HA)

\section{INTRODUCTION}

Mobile IPv6 (MIPv6) (Johnson et al., 2004) protocol has been standardized by the Internet Engineering Task Force (IETF) to address the global mobility of mobile nodes. MIPv6 enables communication session continuity for hosts while they are moving. However, it suffers from long handoff latency, high packet loss ratio and high signaling overhead (Chuang and Lee, 2011).

The Proxy Mobile IPv6 (PMIPv6) (Gundavelli et al., 2008) has been standardized by the IETF NETLMM working group to solve these problems associated to the MIPv6 protocol. PMIPv6 adds two functional entities which are the Mobile Access Gateway (MAG) and the Local Mobility Anchor (LMA). The LMA is similar to the home agent in MIPv6 and its main role is to maintain reach ability to the Mobile Node (MN) address while it moves in the local PMIPv6 domain. The MAG is responsible for detecting the mobile node movements and initiating the required authentication signals with the Authentication, Authorization and Accounting (AAA) server in order to register the MN with the LMA. For the registration to be performed by the MAG, it needs to know the MN's LMA address, MN's network prefix and the allowed address configuration modes. All these information are stored into the AAA server in a centralized or distributed manner (Korhonen and Muhanna, 2008). The salient feature of the PMIPv6 is that it is networkbased, in which, the network detects the node mobility and initiates the required mobility signals, so that, it relieves the mobile node from participating in the handoff process. However, the PMIPv6 still suffers from long handoff latency and packet loss during handoff due to the authentication and registration

Corresponding Author: Adnan J. Jabir, Department of Communication Technology and Networks, Faculty of Computer Science and Information Technology, University Putra Malaysia, 43400 Serdang, Selangor, Malaysia 
signals which are frequently performed during the node mobility (Hwang et al., 2010).

There are many attempts to enhance the PMIPv6 in the literature encompassing different factors. The Fast Proxy MIPv6 (PFMIPv6) (Yokota et al., 2010) protocol which has been standardized by the IETF reduces the handoff latency. However this protocol introduces the problem of false handoff initiation because the serving network predicts which new network the MN will move to (Kim et al., 2010). Most of the handoff related works have been devoted to reduce the packet loss by buffering the incoming packets or to reduce the handoff time by registering the mobile node to the new network in advance. However, There has been insufficient attention has given to reduce the number of steps needed for the authentication and registration processes.

In our knowledge, the only work focused on reducing the number of authentication and registration steps has been done by in (Islam and Huh, 2011). The respected authors of this work have integrated the AAA server with the LMA. Implementing the authentication functions into the mobility server has reduced the number of the required signals. However, it has extended the single point of failure to include both the mobility and the authentication servers, such that if this unit fails, both the mobility and authentication information will be lost. In addition, this method may not work efficiently in a multi mobility anchors environment.

In this study, an enhanced protocol based on PMIPv6 has been proposed and developed analytically to reduce the signaling cost.

this study is organized as follows: we present the PMIPv6 and its signaling as the foundation of the needed related work. Then the proposed signaling is presented. After that, we present the analytical model and the handoff latency analysis with the experimental results. Lastlt, we present our discussion and conclusion.

Related work: The analysis of the existing research has been done with the main intention of providing an absolute deliberation of the standard dominance.

Overview of PMIPV6: The main idea behind PMIPv6 is to relieve mobile nodes from participating in any IPlayer mobility signalling assuming that the mobility functions are performed by the network itself. PMIPv6 supports mobility within a localized area which is defined as the PMIPv6 domain, in which, the mobile node retains its IP-address while moving and the network is responsible for tracking its location. It is a network-based mobility management protocol derived from MIPv6 and it reuses the Home Agent (HA) concept but by adding new entities for performing the mobility signals on behalf of mobile nodes (Islam and Huh, 2011; Kim et al., 2008).

Proxy mobile IPv6 protocol (Gundavelli et al., 2008) is standardized by IETF as an extension to MIPv6 protocol to provide network mobility management for a mobile node in a topologically localized domain without requiring the mobile node to participate in any mobility signaling. The new added functional entities to PMIPv6 are Mobile Access Gateway (MAG) and Local Mobility Anchor (LMA). Fig. 1 illustrates the network entities in the PMIPv6 protocol and their operating mechanism in the local mobility domain (Kong et al., 2008).

The main role of MAG, which is deployed on the access router, is to detect the mobile nodes movement and to initiate the required mobility signals with LMA on behalf of the mobile node. The second function of MAG is to establish a tunnel with LMA to enable the mobile node to maintain an address from its home network prefix. The main responsibility of the LMA is to maintain the reach ability to the mobile node address while it moves within the PMIPv6 domain.

LMA is similar to the home agent in MIPv6, but it has some additional capabilities to support PMIPv6. To perform its role, it maintains a binding cache entry for each registered mobile node, but differs from that of the home agent by its additional fields including mobile node identifier and MN home network prefix. The entry also contains a flag for indicating a proxy registration and an identifier for the interface of MAG-LMA tunnel.

Message flow of PMIPV6: The mobility scenario in PMIPv6 can be described as follows: when an MN enters its PMIPv6 domain and upon the completion of access authentication. The serving network assigns a unique home network prefix to the node. This network prefix is unique for each mobile node and will be retained wherever it moves inside the PMIPv6 domain. Therefore, the mobile node is given the impression that the entire PMIPv6 domain is its home network. Hence, there is no need for the mobile node to reconfigure a care of address every time it moves. The MN attachment, authentication and registration operations in the PMIPv6 are shown in Fig. 2 with their required message flow (Kong et al., 2008).

Each step of the PMIPv6 is described as follows (Kong et al., 2008):

Steps1 and 2: Once the MAG detects an attached MN, it sends an authentication query to the AAA server which contains the MN Identifier (MN-ID). This query is performed by the MAG to make sure that the MN is authorized to access the network. 


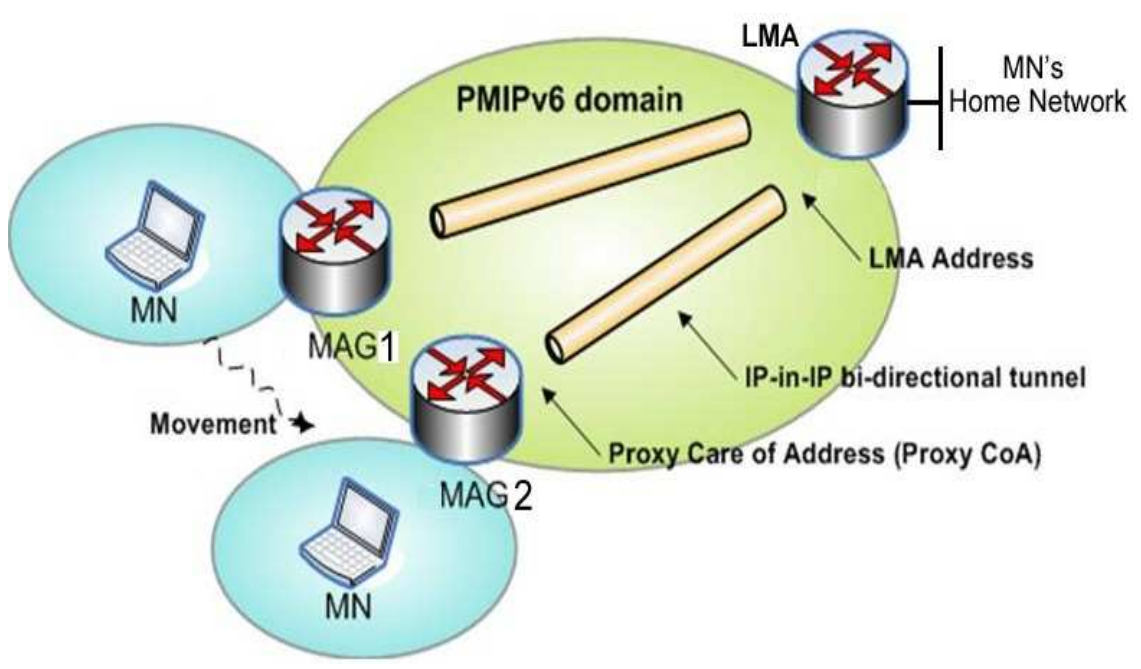

Fig. 1: Overview of PMIPv6 (Kong et al., 2008)

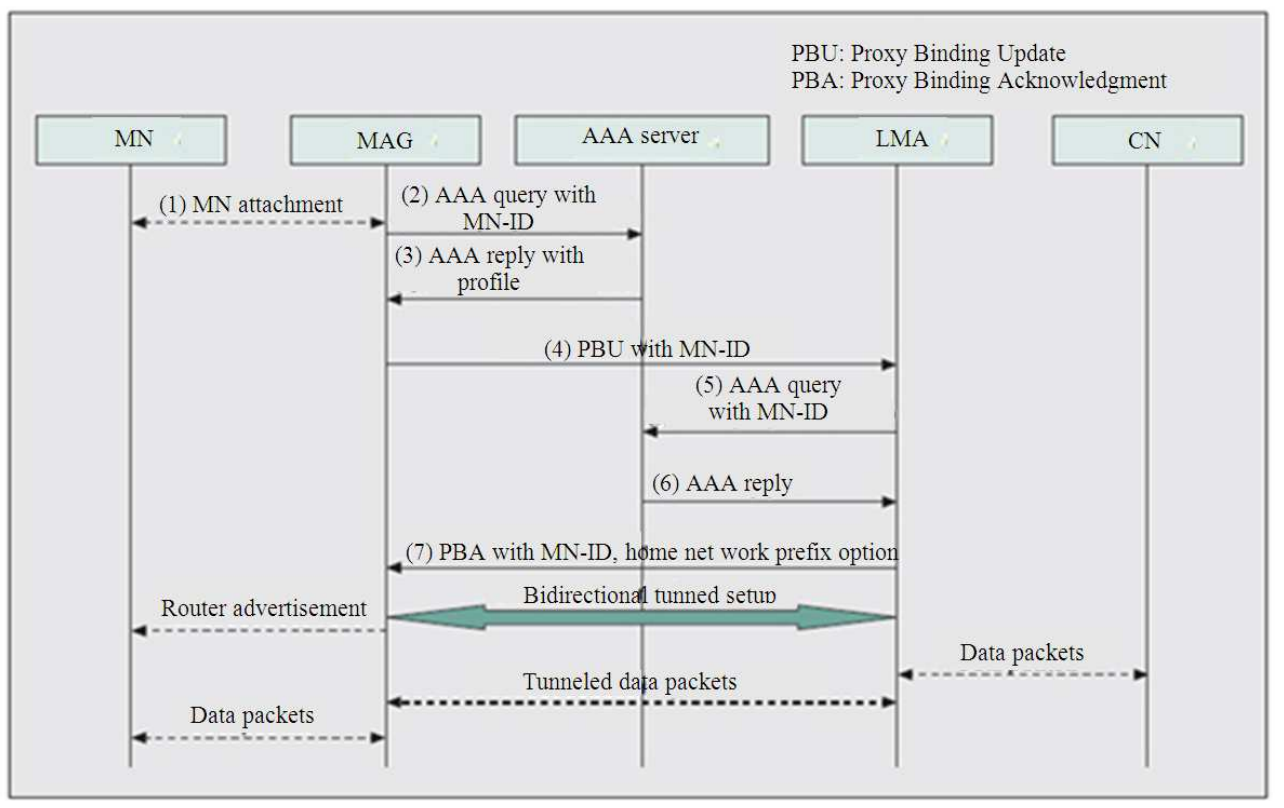

Fig. 2: PMIPv6 message flow (Kong et al., 2008)

Step3: The AAA server checks the MN-ID and replies by sending back the MN's profile if the access authentication is successful. The MN's profile contains the needed MN's information such as MN-ID, MN's corresponding LMA address and the address configuration scheme.

Step 4: When the requesting MAG receives a reply indicative successful authentication from the AAA server, it sends a Proxy Binding Update (PBU) message to the MN's LMA to register or to update the MN's information in the corresponding LMA.

Steps5 and 6: When the LMA receives the PBU message, it queries the AAA server to ensure that the requesting MAG is trusted. If it gets a positive reply from the AAA, it accepts the PBU message.

Step 7: The last step in the authentication and registration process is performed by the LMA through the sending of a Proxy Binding Acknowledgement (PBA) 
message. This message contains the MN's home network prefix to be used by the MN to maintain its IPv6 address. Then the LMA sets up a bi-directional tunnel with the corresponding MAG to be used for routing the traffic to and from the $\mathrm{MN}$.

When the MAG gets the required MN's information from the PBA message, it sends a Router Advertisement (RA) message to the mobile node including the home network prefix. The MN uses this prefix to configure its home address depending on the supported mode for the address configuration mode which is either in a stateful or stateless mode.

From Fig. 2, it can be seen that the AAA server is accessed extensively. The analysis of these accesses has motivated this research. Redundancy of the signaling has been utilized as the foundation of the proposed work.

The proposed I-PMIPv6: The main idea of the proposed model is to reduce the redundant signaling in the authentication and registration processes of PMIPv6 and the involvement of the AAA server in the registration process. As deliberated previously in this study, the AAA server is always accessed by both the MAGs and the LMAs for authentication queries during the registration of the mobile nodes. In PMIPv6, when the MAG sends an AAA query to the AAA server; its consecutive operation is constrained by the obligatory wait of the AAA reply before sending the PBU to the LMA as shown in Fig. 2 (steps 2, 3 and 4 respectively). In the proposed I-PMIPv6, these signaling features are redesigned to achieve intended task with much reduced accumulated signaling. This will reduce the number of signals from a 3 signals to only 2 signals which are MAG $\rightarrow$ AAA $\rightarrow$ LMA. Therefore, the MAG's authentication signals which are initiated by the LMA Fig. 2 (steps 5 and 6) become redundant and insignificant signals. This is because the LMA will receive the PBU from the AAA server which has already authenticated both the $M N$ and the requesting MAG. As a result, the total signaling needed for the authentication and registration cycle is reduced from 6 signals to 3 signals only as shown in Fig. $3 a$ and $b$.

In the proposed I-PMIPv6, when the AAA server receives an AAA request from a MAG; it performs the needed authentication depending on the MN-ID and then directly sends a PBU request to the LMA containing the MAG address and the MN-ID. Once the LMA receives the PBU from the AAA server, it will directly reply by a PBA message to the requesting MAG after performing the needed registration functions and then starts creating the required bi-directional tunnel with the requesting MAG.

The mobile node attachment, authentication and registration steps in the proposed I-PMIPv6 are shown in Fig. 4 with their required message flow. Each step is described as follows:

Steps 1 and 2: Once the MAG detects an attachment of an $\mathrm{MN}$, it sends an authentication query to the AAA server containing the MNID. This query is performed by the MAG to make sure that the $\mathrm{MN}$ is authorized to access the network

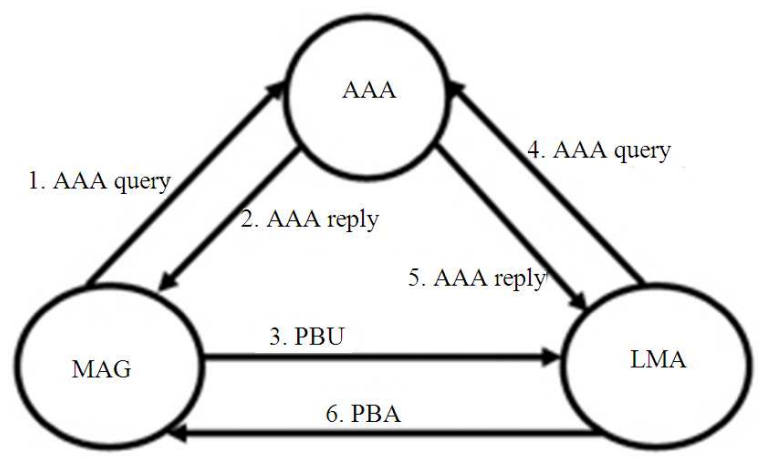

(a)

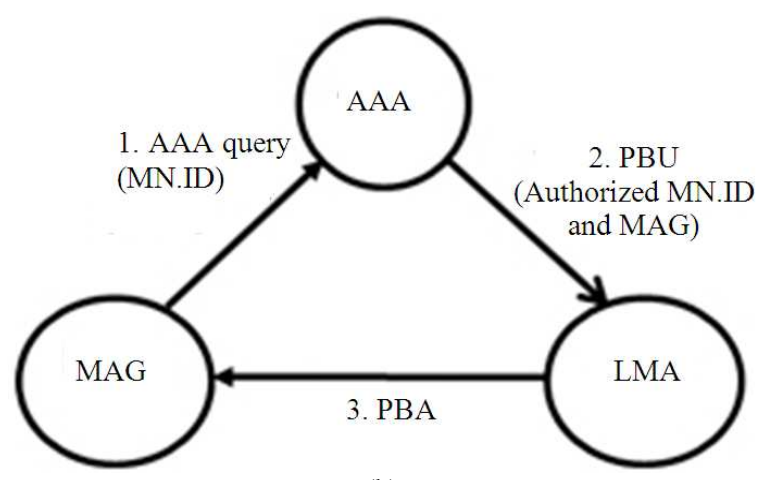

(b)

Fig. 3: The authentication and registration cycle (a) PMIPv6, (b) The proposed I-PMIPv6

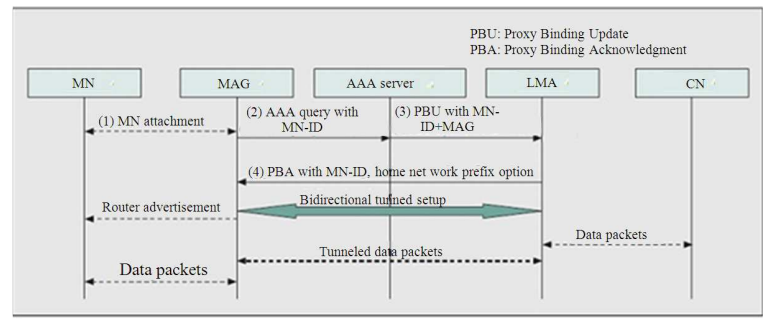

Fig. 4: I-PMIPv6 message flow 


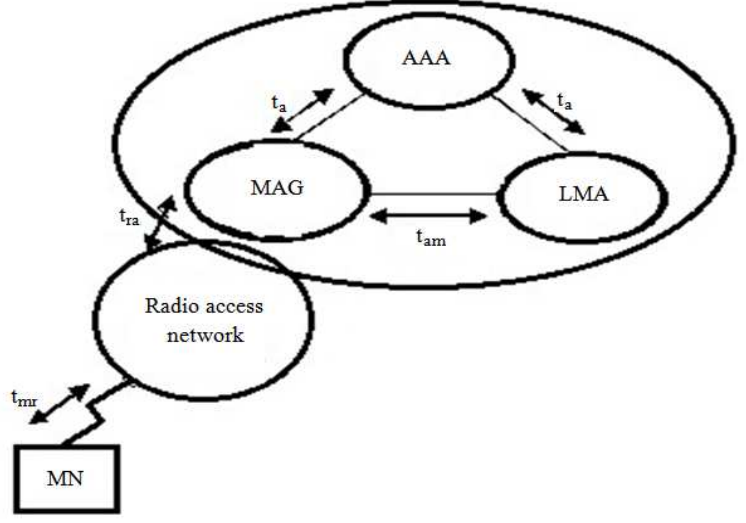

Fig. 5: A simple analytical model for performace analysis

Step 3:

After a successful access authentication, the AAA server sends a PBU request to the LMA on behalf of the requesting MAG. It sends the needed information including MN-ID and the MAG address.

Step 4: Once the LMA receives the PBU message from the AAA server, it sends a PBA message to the requesting MAG (which is specified by the PBU request) including the MN's home network prefix option and sets up a route for the MN's home network prefix over the tunnel to the MAG. In this case, there will be no need for the LMA to perform the MAG authentication as both the requesting MAG and the MN have already been authorized by the AAA server

The analytical model: The simple analytical model used by (Kong et al., 2008) has been adopted with the respective needed extensions as shown in Fig. 5. The model serves as the underlying platform for the handoff latency for both PMIPv6 and I-PMIPv6 analysis.

For the comparison purposes, we use the same notations and assumptions which are used by (Kong et al., 2008) as follows:

$\mathrm{t}_{\mathrm{mr}}=$ The delay time required to send a packet through a wireless link between the $\mathrm{MN}$ and the access network (Access Point-AP)

$\mathrm{t}_{\mathrm{ra}}=$ The delay time between the AP and the MAG.

$t_{a m}=$ The delay time required to send a packet through wired link between the MAG and its LMA

$\mathrm{t}_{\mathrm{a}}=$ The delay time required to send a packet through wired link between the MAG and the AAA server or between the LMA and the AAA server.
Handoff latency: The IP Handoff Latency ( $\mathrm{HL}_{\mathrm{IP}}$ ) is defined as the time needed for the MN to change its point of attachment from one network connection to another. More specifically, it is the time between the moment of completing the layer 2 handoff and the moment of receiving the first data packet from the new point of attachment (Kong et al., 2008; Dinakaran and Balasubramanie, 2012). It can be expressed as follows Eq. 1:

$\mathrm{HL}_{\mathrm{IP}}=\mathrm{TMD}+\mathrm{TDAD}+\mathrm{TAAA}+\mathrm{TREG}$

Where:

TMD $=$ The movement detection time

TDAD $=$ The address configuration time

TAAA $=$ The authentication, authorization and accounting time

TREG $=$ The location registration time

One of the salient features of PMIP6 is that it does not require movement detection and DAD except when the MN first enters a PMIPv6 domain. As a result, the handoff latency of the PMIPv6 can be expressed as the sum of TAAA, TREG and the packet transmission delay between the MN and the MAG as follows Eq. 2 and 3 (Kong et al., 2008):

$\mathrm{HL}_{\mathrm{PMIPv} 6}=\mathrm{TAAA}+\mathrm{TREG}_{\mathrm{PMIPv} 6}+\mathrm{t}_{\mathrm{mr}}+\mathrm{t}_{\mathrm{ra}}$

Where:

TAAA $=2 \times 2 t_{a}$; two signals between the MAG and the AAA server and the other two signals are between the LMA and the AAA server

$\mathrm{TREG}_{\mathrm{PMIPv} 6}=2 \mathrm{t}_{\mathrm{am}}$; for PBU and PBA messages

$\mathrm{t}_{\mathrm{mr}}+\mathrm{t}_{\mathrm{ra}}=$ The wireless packet transmission delay between the MN and the MAG

Finally:

$\mathrm{HL}_{\mathrm{PMIPv}}=4 \mathrm{t}_{\mathrm{a}}+2 \mathrm{t}_{\mathrm{am}}+\mathrm{t}_{\mathrm{mr}}+\mathrm{t}_{\mathrm{ra}}$

In the proposed I-PMIPv6, the authentication signals are reduced to two signals only (one from MAG to AAA and the other from AAA to the LMA) and the registration time is also reduced to only one signal (for sending a PBA message from an LMA to a MAG) as the PBU has already been considered as one of the authentication signals from the AAA server to the LMA. Hence, the handoff latency delay for I-PMIPv6 is expressed as follows Eq. 4 and 5:

$\mathrm{HL}_{\mathrm{I}-\mathrm{PMIPv} 6}=\mathrm{TAAA}+\mathrm{TREG}_{\mathrm{I}-\mathrm{PMIPv} 6}+\mathrm{t}_{\mathrm{mr}}+\mathrm{t}_{\mathrm{ra}}$ 
Am. J. Applied Sci., 9 (4): 535-541, 2012

$\mathrm{HL}_{\mathrm{I}-\mathrm{PMIPv} 6}=2 \mathrm{t}_{\mathrm{a}}+\mathrm{t}_{\mathrm{am}}+\mathrm{t}_{\mathrm{mr}}+\mathrm{t}_{\mathrm{ra}}$

From Eq. 3 and 5, it can be abstracted that the IPMIPv6 has better result in a handoff latency time in comparison to the PMIPv6 protocol by the number of reduced value associated to individual factors constituting the summation.

\section{MATERIALS AND METHODS}

In this study, the simple analytical model which is used by (Kong et al., 2008) is adopted with the respected extensions to compare the performance of our proposed I-PMIPv6 with the PMIPv6. The Eq. 3 and 5 are used to calculate the handoff latency for both protocols. We start by measuring the impact of the wireless delay on the handoff latency time by changing its value and considering the wired link as a constant value. Then we compute the impact of the wired link delay on the handoff latency time. These two calculations are used to show the effect of reducing the number of signals on the handoff latency time.

\section{RESULTS}

The numerical results based on the derivations in Eq. 3 and 5 is presented here. In ensuring a level comparative platform, the assumptions used by (Kong et al., 2008) have been used in the research.

They are as follows:

$\mathrm{t}_{\mathrm{a}}=3 \mathrm{~m} \mathrm{sec} ; \mathrm{t}_{\mathrm{am}}=10 \mathrm{~m} \mathrm{sec} ; \mathrm{t}_{\mathrm{mr}}=10 \mathrm{~m} \mathrm{sec} ; \mathrm{t}_{\mathrm{ra}}=2 \mathrm{~m} \mathrm{sec}$

Impact of wireless link delay: The Fig. 6 show how does the wireless link delay time affects the handoff latency for both the PMIPv6 and the proposed IPMIPv6. It can be seen that with the increment of the wireless link delay, the handoff latency is increased for both PMIPv6 and I-PMIPv6. However, the handoff latencies of PMIPv6 are significantly larger than that of I-PMIPv6. This is because the time required for the authentication and registration is less for I-PMIPv6 (TAAA + TREG) which is a constant term here.

Impact of wired link delay: The Fig. 7 shows the impact of the wired link delay time on the handoff latency for both PMIPv6 and I-PMIPv6. It can be seen that with the increment of the wired link delay, the handoff latency is increased for both PMIPv6 and IPMIPv6. However, the handoff latencies of PMIPv6 are significantly larger than that of I-PMIPv6. This is distinctly observed because the total number of the required signals for the authentication and registration are decreased to $50 \%$ for the I-PMIPv6.

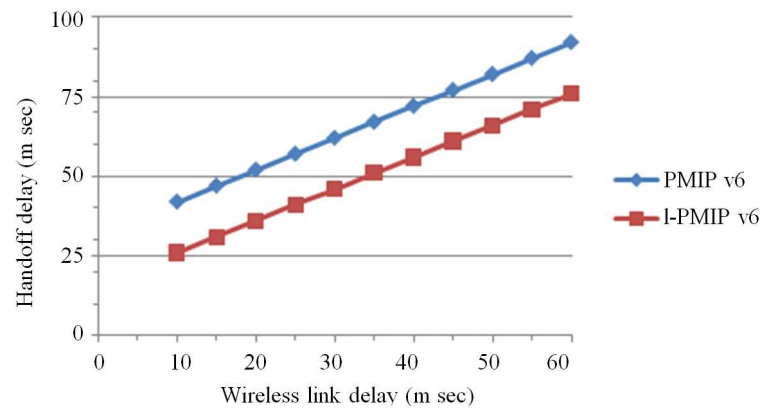

Fig. 6: A comparsion between PMIPv6 and I-PMIPv6 on the impact of the wireless delay.

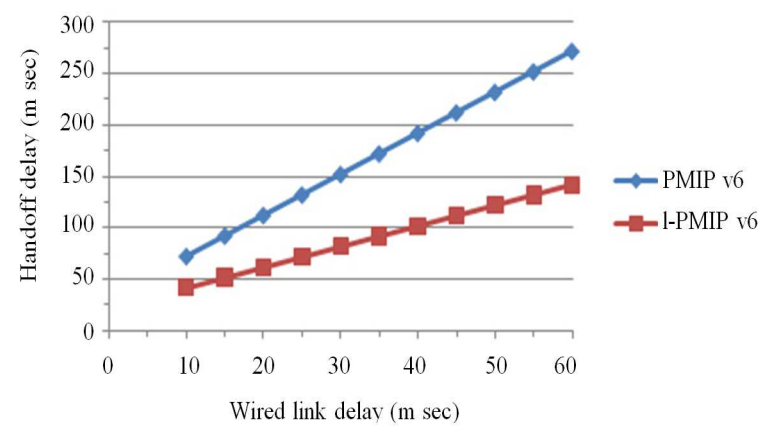

Fig. 7: A comparsion between PMIPv6 and I-PMIPv6 on the impact of the wired delay

\section{DISCUSSION}

The optained results which are presented in Fig 6 and 7 show that the proposed I-PMIPv6 outperforms the PMIPv6 in terms of the handoff latency time. We calculate the handoff latency time depending on different wireless and wired delay time. In both cases, the I-PMIPv6 shows lower latency time in comparison with the PMIPv6 because the former offers lesser registration and authentication signaling. It is also necessory to notice that the handoff latency is highly affected by the wired link delay as shown in Fig 7. This is reasonable because the authentication and the registration processes are performed on the wired lines between the MAGs, AAA and the LMA.

\section{CONCLUSION}

In this study, the I-PMIPv6 which enhances the PMIPv6 protocol signaling cost was deliberated. TheIPMIPv6 integrates the authentication and the registration signals to reduce the handoff latency time. The obtained performance analysis demonstrates the superiority of the I-PMIPv6 via the low singaling overhead and the reduction of the load on the AAA 
server. A pertinent attribute of the I-PMIPv6 is that there will be two types of PBU, one is generated by the AAA server to the LMA for the MN registration and the other one is generated by the MAG to the LMA for the MN's de-registration. Therefore, the deregistration PBU can be sent directly from the MAG to the LMA without any need to go through the AAA server. An identified implementation consideration for the proposed method is the structure of the PBU message. The PBU message which is requested by the AAA should be extended to include both the MAG and the MN-ID. The future work consist the implementation of the I-PMIPv6 protocol and to embark on the cooperation between the PFMIPv6 and the proposed I-PMIPv6.

\section{REFERENCES}

Chuang, M.C. and J.F. Lee, 2011. FH-PMIPv6: a fast handoff scheme in proxy mobile IPv6 networks. Proceedings of the IEEE International Conference on Consumer Electronics, Communications and Networks, Apr. 16-18, IEEE Xplore Press, XianNing, pp: 1279-1300. DOI: 10.1109/CECNET.2011.5768193

Gundavelli, S., K. Leung, V. Devarapalli, K. Chowdhury and B. Patil, 2008. Proxy Mobile IPv6. RFC 5213 (Proposed Standard).

Dinakaran, M. and P. Balasubramanie, 2012. An efficient hand-off mechanism for vehicular networks. J. Comput. Sci., 8: 163169. DOI: 10.3844 jessp.2012.163.169

Hwang, H., J.H. Kim, J.S. Lee and K.G. Lee, 2010. Fast handoff scheme using multicast group for intradomain in PMIPv6 networks. Proceedings of the 7th IEEE Conference on Consumer Communications and Networking, Jan. 9-12, IEEE Xplore Press, Las Vegas, NV., pp: 1-2. DOI: 10.1109/CCNC.2010.5421681
Islam, M.M. and E.N. Huh, 2011. Sensor Proxy Mobile IPv6 (SPMIPv6)-A novel scheme for mobility supported IP-WSNs. Sensors, 11: 1865-1887. DOI: 10.3390/s110201865

Johnson, D., C. Perkins and J. Arkko, 2004. Mobility Support in IPv6.

Kim, B., J. Yang and I. You, 2008. A survey of NETLMM in all-IP-based wireless networks. Proceedings of the International Conference on Mobile Technology Applications System, (ICMTAS' 08), ACM, USA., DOI: 10.1145/1506270.1506348

Kim, M.S., S.K. Lee, D. Cypher and N. Golmie, 2010. Fast handover latency analysis in proxy mobile IPv6. Proceedings of the IEEE Global Telecommunications Conference, Dec. 6-10, IEEE Xplore Press, Miami, FL., pp: 1-5. DOI: 10.1109/GLOCOM.2010.5684107

Kong, K.S., W. Lee, Y.H. Han, M.K. Shin and H.R. You, 2008. Mobility management for all-IP mobile networks: Mobile IPv6 Vs. proxy mobile IPv6. IEEE Wireless Commun., 15: 36-45. DOI: 10.1109/MWC.2008.4492976

Korhonen, J. and A. Muhanna, 2008. Policy Profile and AAA interfaces requirements for PMIPv6. The IETF Trust.

Yokota, H., K. Chowdhury, R. Koodli, B. Patil and F. Xia, 2010. Fast handovers for proxy mobile IPv6. IETF Trust. 